\title{
STEREOPHONIC SOUND RECORDING, REPRODUCTION AND BROADCASTING
}

$I^{\mathrm{T}}$ $T$ is commonly remarked, with much truth, that engineering is impatient of scientific understanding ; history can cite repeated instances of engineering enterprise plunging ahead and producing devices of great efficiency and utility, which only afterwards have been blessed by scientific theory when this has shown them to have reached near-theoretical limits. For this, the engineer needs no apology. His first concern is not analysis but synthesis; his purpose is a social one, and he must for ever be content with compromise, drawing upon such science as may exist, using judgment and even guesswork to satisfy his customers.

This has been true of most branches of the electronics industry; radio, radar, television and now 'hi-fi' and stereophony are triumphs not primarily of science but of technology.

Stereophonic recording, reproduction and broadcasting, the subject of a Convention held under the auspices of the Institution of Electrical Engineers on March 19 and 20, represents one of the latest catchpurses to tickle the public fancy. Such conceits spread quickly, perhaps we might unkindly say, first among those who lust after the latest gadgets; but if this newest branch of the radio and gramophone industry is thus encouraged, there is no doubt that musical education will also benefit eventually. But the practical systems of stereophony are as yet imperfect, and there is room for ingenuity and inventiveness - and, in my opinion, more room now for science.

This was perhaps the most disappointing aspect of the Convention, that it was concerned almost entirely with techniques; with practical problems of recording head design, with standardizing the grooves in gramophone records, with magnetic-tape machine design for easy operation and low cost, with techniques of compatibility whereby stereophonic (twin-track) signals may be used by those unfortunates who possess conventional one-loudspeaker sets-but almost nothing was heard concerning the theoretical basis of stereophony, nor of the unsolved problem of binaural hearing. For we still do not fully understand directional hearing, either psychologically or physiologically.

The opening paper, by Mr. T. Somerville, rightly emphasized the pioneer work of Blumlein, some thirty years ago. Blumlein's name is specially associated with systems of stereophony which use coincident microphones; these are not spaced apart in the studio, but have their directional characteristics angularly spaced, such that a sound reaching them sets up two signals with different amplitudes.

It would have helped the Convention if, at this early stage, some explanation of the essential theory of stereophonic hearing had been presented-aided by simple geometric diagrams. In this way we might have avoided several confusions which arose later. For example, simple geometry readily shows how, in Blumlein-type systems, the amplitude-difference at the two microphones becomes converted into a time-difference between the two ears of a listener at the receiving end. Of course, our sense of direction does not entirely depend upon the binaural time- interval but upon other sensory clues as well - but it is very important.

Mr. F. H. Brittain, who followed with a paper comparing normal directional hearing with that provided by the twin loudspeakers of stereophony systems, valiantly tried to clear up certain misconceptions. Thus he stressed that the signal from any one microphone in the studio, fed to one loudspeaker in your living-room, reaches both your ears. The facts that you have two ears and that usually two microphones are used are not related-- the microphones do not in any sense "simulate your two ears" (one later speaker had the good grace to apologize for a slide, which showed a symbolic human head wrongly placed between two spaced microphones). Again, Mr. Brittain pointed out the distinction between inter-aural time-intervals (maximum, about 500 microseconds in normal conditions) and intersource, loud-speaker, time intervals (which may be several milliseconds). It is only the latter interval which concerns the so-called 'Haas effect', an effect which is of some importance for off-axis listening in stereophony. Thus if you sit well to one side of the room, the sound from one loudspeaker may reach both your ears 4-5 milliseconds before the sounds from the other ; in such circumstances, the sounds at each ear may fail to fuse, subjectively, and the illusion of spatial distribution is shattered.

In normal life our sense of sound direction arises from several acoustic clues; in particular, differences in inter-aural time, intensity and in minor sound structure. But such differences alone cannot give an image outside the head-they produce right/left shifts only. We need, further, the variations of these differences with head turning (aural-kinæsthetic association). The only detailed references to these basic facts were made by Mr. Brittain, who outlined the difficulties involved in producing these desired inter-aural characteristics in the sound field from two spaced loudspeakers. He stressed that, ideally, we need loudspeakers with directional lobe-patterns of a special shape and which vary with frequency; further, it would greatly help if the time-delay of sounds emitted from each loudspeaker could be varied suitably with angle. We could well have profited by a good deal more physical and psychological background to this Convention.

The remaining papers were technical. One by Mr. G. F. Dutton and another by Messrs. A. D. Burt and D. R. Andrews were concerned with magnetic tape recording. The first outlined the commercial possibilities of $7 \frac{1}{2}$ in./sec. tape records and reproducers, which now can be operated by people who are not mechanically minded. Tape records can be made with wide frequency-bands, with negligible 'print-through' between layers when stored and, most important, very low 'cross-talk' between the adjacent tracks of the two channels. This latter point was much discussed, and clearly it is a troublesome aspect of technique.

Mr. Dutton also outlined the problems of reducing the cost of stereophony on tape records, which relate to the use of slower speeds, involving better head resolution, smoother tapes and narrower tracks. 
Very similar points were raised by the authors of the second paper, who dealt specially with the head design and with easily handled, quick-change tape 'cartridges'.

The final paper on the first day of the Convention, presented by Mr. J. Moir, discussed cinema stereophony. It is characteristic of cinema systems that they have used $3,4,5$ or even 6 loudspeakers, as opposed to the usual pair of domestic systems. Now with two microphones, only two-point samples of a continuous sound field in the studio are taken, so that the two spaced loudspeakers in your living-room set up a kind of diffraction pattern; this is perhaps more or less correct if you sit in the middle, but not if you sit to one side. Cinema audiences, however, occupy a wide angle, giving corresponding difficulties. One might have hoped that this greater number of spaced microphones would be used to give a more accurate simulation of the studio sound field, but Mr. Moir pointed out that studio personnel have not, in the main, understood the true problem. Most film recordings have used single-track technique, merely switching the sound image about the screen to be roughly coincident with the photographic image, by potentiometer control of the recording level from each microphone: a difficult proceeding if two actors speak simultaneously.

An evening session was devoted to broadcast stereophony. Mr. D. E. L. Shorter stressed the difficulties of 'compatibility'-conventional one-channel reception of an intended stereophony broadcast--and the technical difficulties of modulating a single carrier wave by two sources. Dr. W. S. Percival described an ingenious stereophonic system in which the two channels convey $(a)$ the main acoustic content, (b) the acoustic direction clues, the latter requiring very low information rates indeed; he quite rightly observed that our sense of direction is most sensitive to transient sounds, and his system takes maximum notice of these.

Two papers were delivered on the second day dealing with disk recording. Mr. Dutton referred to internationally agreed standards, both of cutting and pick-up techniques. The most-favoured system, called the $45 / 45$ system, uses orthogonally placed needles at $45^{\circ}$ to the vertical. For best performance, minimum wear, cross-talk, etc., fully mechanical standards have now been set up. Great attention is also being paid to improvements in cutter and pick-up design, for better reproduction and easier manufacture, a topic dealt with in some detail by later speakers.

The Convention closed with a lively discussion, admirably refereed by Mr. Moir, which provided as much interest as some of the main proceedings. Perhaps the Institution of Electrical Engineers can be persuaded to present another Convention later. dealing with principles and fundamental problems rather than so much with design of apparatus. Engineers have gone a long way in this field on inadequate theory, or on none at all. This Convention showed the need now to be pressing.

Colin CherRy

\section{INDUSTRIAL PHOTOGRAPHIC AND TELEVISION EXHIBITION}

$\mathrm{T}$ HE Industrial Photographic and Television Exhibition, which was held at the Royal Albert Hall during April 20-24, was sponsored and organized by the Financial Times. It was stated that "the object of the exhibition was to show executives the way in which photography can aid them in many stages of the industrial process or commercial activities".

Throughout the duration of the exhibition a number of lectures were given each day on the use of photography and television in the factory, the office, training and advertising.

The most obvious use of photography is its ability to record events for future reference. A photograph can store, in one instant of time, much more information than an observer could absorb-information the importance of which may not even be appreciated at the time. Such records can be examined in detail and at leisure at a later date. They can also be compared with others taken at different times, in order to find similarities and differences, as in astronomy.

In addition, photography can extend the field of human observation. If an event lasts only a fraction of a second, high-speed photography can be used to slow the action down. On the other hand, some changes are difficult to follow because they occur so slowly. By using time-lapse photography, the timescale can be compressed and the whole cycle of action presented in a few minutes or less.

Furthermore, sensitized photographic materials are sensitive to many forms of radiation to which the eye does not respond in a useful manner, such as infra-red and ultra-violet radiation, $\alpha_{-}, \beta-, \gamma-$ and $\mathrm{X}$-rays and atomic particles.

$X$ - and $\gamma$-rays are used in radiography, which is a non-destructive method of examining the internal structure of a wide range of objects, including human beings. From X-ray and electron diffraction patterns information can be obtained about the fine structure of materials, and in the electron microscope the use of an electron beam enables greater resolution to be obtained than with the optical microscope.

Special emulsions have been developed to help the nuclear physicist record the tracks of particles such as electrons, protons, mesons, etc., moving at high speeds. From these records he gains knowledge of atomic and nuclear structure.

The most striking feature of the exhibition was a large display of excellent black-and-white and coloured prints illustrating the uses to which photography could be put in industry, research, documentation and advertising.

Among these was a set of prints taken by reflected polarized light showing the growth of magnetic domains on the surface of a metal with increasing magnetic field (International Computers and Tabu. lators, Ltd.).

Examples of high-speed photography included a study of spray formation from high-pressure jets (Babcock and Wilcox, Ltd., and John Thornycroft, Ltd.). Another series of photographs illustrated a method of demonstrating fluid-flow by recording the paths taken by numerous small air bubbles suspended in a liquid. The apparatus was illuminated from one side by a flash bulb through a narrow slit so that a 